\title{
Untersuchung des Einflusses von iatrogenen Schädigungen auf Marknägel
}

\author{
Bock S.H., Bechtold J.E.*, Kraft M., Boenick U. \\ Institut fur Mikrotechnik und Medizintechnik, Technische Universităt Berlin \\ *Orthopaedic Biomechanics Laboratory, Midwest Orthopaedic Research Foundation (MORF) at the \\ Hennepin County Medical Center, Minneapolis, Minnesota, V.St.A.
}

\section{EINLEITUNG}

Marknägel werden zur internen Fixierung von Brilchen der langen Rohrenknochen benutzt. Dabei muß der Nagel physiologische Lasten durch Biegung, Druck (d.h. Verkürzung) und Verdrehung aufnehmen. Der Rotationsschutz zur notwendigen Drehmomentubertragung, sowie auch die Längenkontrolle, wird ublicherweise durch eine Verriegelung des Nagels mit Schrauben oder Bolzen erreicht, die in proximalen und distalen Querbohrungen des Marknagels sitzen. Vor dem Einsatz der Verriegelungsschrauben durch Knochen und Implantat muß der den Nagel umgebende Knochen durchbohrt werden.

Für die proximale Verriegelung stehen je nach Nagelhersteller verschiedene Lehren zur Verfügung, die eine präzise Führung des Bohrers gewährleisten. Da ein biegsamer Nagel jedoch beim Einschlagen dem Markraum folgt, ist man beim distalen Verriegeln auf eine durchleuchtungsgestützte Freihandtechnik angewiesen, wobei eine Kollision des Bohrers mit dem Rand des Verriegelungsloches des Marknagels ein oft beobachtetes Problem ist. Da bei den Querbohrungen der Marknagel ohnehin die geringste Festigkeit hat, ist eine in diesem Bereich beigebrachte Schädigung von erhöhter Brisanz. Tatsächlich treten Nagelbriuche zumeist im Bereich der distalen Verriegelungsöffnungen des Marknagels auf [1].

Eine Vorstudie am Orthopaedic Biomechanics Laboratory klärte zunächst den Einfluß einer schweren (und deshalb unrealistischen) Kerbe in diesem Bereich auf solche Bauteile. Alle hierbei geschädigten Proben versagten in den durchgefuhrten Dauerschwingversuchen erheblich eher, als die ungekerbten Vergleichsproben. Die Ergebnisse gaben den Anlaß zur Suche nach einer differenzierteren, den operativen Gegebenheiten angepaßten Methode [2].

\section{MATERIALIEN}

Zum Ausschluß des Einflusses der Geometrie der unterschiedlichen verwendeten Marknägel wurden nur gleiche, runde Rohre mit $9 \mathrm{~mm}$ Außendurchmesser und $1,5 \mathrm{~mm}$ Wandstärke verwendet. Alle 36 Proben hatten die gleiche 5-mm-Verriegelungsbohrung. Es wurden Rohre aus austenitischen Stahl (316L und 22-13-5) sowie einer Titanlegierung (Ti-6Al-4V), wie sie für Marknägel verwendet werden, untersucht.
Bei 316L handelt es sich um einen kohlenstoffarmen eisenbasierenden Stahl der aus dem 302 (in Deutschland besser bekannt als $34 \mathrm{CrNi} 188$ oder auch V2A) entwickelt wurde. Er enthält $2-3 \%$ Molybdän zur Erhöhung der Lochfraßresistenz und wird zur Verfestigung vor der Verarbeitung kalt gewalzt. Zusätzlich wurde $316 \mathrm{~L}$ im kugelgestrahltem Zustand getestet, um die Effektivităt dieser zusătzlichen Oberflächenhärtung zu untersuchen.

Der Stahl 22-13-5 ist eine austenitische Eisenbasislegierung, mit einer ăhnlichen Zusammensetzung wie 316L. Auch sie wird vor der Anwendung kalt verfestigt, und enthält weniger Nickel und Molybdän als 316L.

Die am häufigsten verwendete Titanlegierung ist Ti-6Al-4V; eine $\alpha-\beta$-Titanlegierung in der das kubisch raumzentrierte Vanadium die normalerweise bei Titan erst ab $883^{\circ} \mathrm{C}$ existierende $\beta$-Phase schon bei Raumtemperatur als. Zweitphase stabilisiert. Die getesteten Titanproben trugen zudem eine verdickte Oxidschicht (nach AMS 2488C, auch Tiodizing ${ }^{\mathrm{TM}}$ genannt). Dieser Prozeß, bei dem eine etwa $1 \mu \mathrm{m}$ dicke Oxidschicht entsteht, soll das Zerstören der ansonsten nur etwa 10nm dicken Passivschicht erschweren.

Wie bei krz-Eisen $(\alpha)$, so zeigt sich auch bei der getesteten $\alpha$ - $\beta$-Titanlegierung eine Ermüdungsgrenze, die man dagegen bei den austenitischen Stählen (kfzEisen $(\gamma))$ nicht beobachtet.

Alle verwendeten Proben wurden von Marknăgelherstellern geliefert, und entsprachen in Zusammensetzung und Bearbeitung genau den für Marknägel verwendeten Materialien. Wie schon erwähnt, waren die Proben in ihren Abmessungen identisch, um einen Vergleich der erreichten Lastwechsel zu ermoglichen.

\section{METHODE}

Die Belastungen, denen ein Marknagel ausgesetzt ist, sind nicht nur stark von Gewicht und Mobilităt des Patienten abhängig, sondern auch vom Zustand des umgebenden Gewebes und dem Sitz im Knochen. Vereinfachte mechanische Modelle berlicksichtigen nicht den erheblichen Einfluß des am Knochen ansetzenden weichen Gewebes und können deshalb nur eingeschränkt als Berechnungsgrundlage für die Beanspruchung solcher Implantate dienen. $\mathrm{Da}$ Marknägel nach der Knochenheilung entfernt werden, 
wird allgemein eine Lastspielzahl von 500.000 Zyklen als ausreichend betrachtet.

Für die realitătsnahe und reproduzierbare Simulation iatrogener Schădigungen von Marknăgeln wurde eine Vorrichtung entworfen, bei der die in vitro auftretende

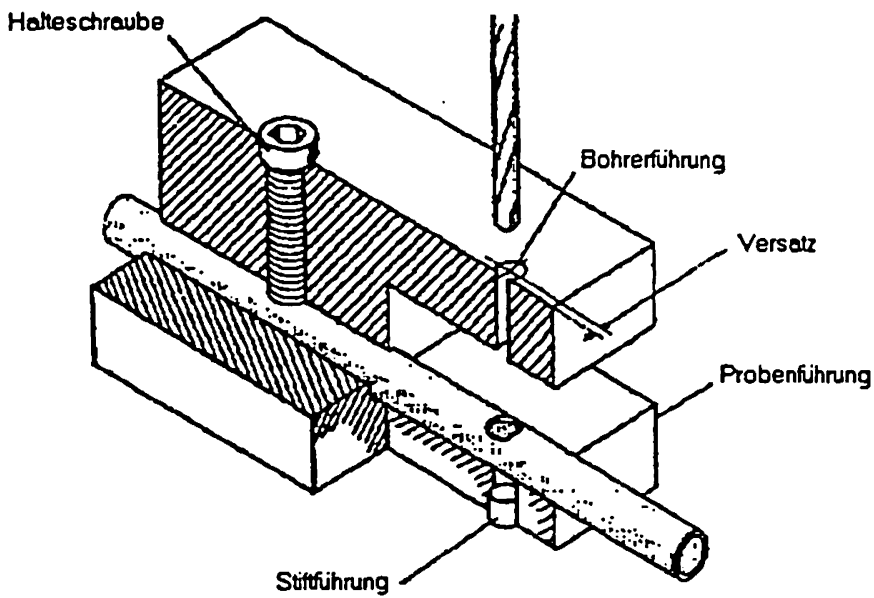

Flexion des Bohrers durch einen Spalt zwischen Probenhalter und Bohrerfuhrung erreicht wurde.

\section{Abb. 1: Entwurf der Schädigungsvorrichtung}

Die Probe wird durch einen Stift in der Halterung positioniert und mit einer Schraube fixiert. Die Bohrerfuhrung zeigt mit ihrer Mittellinie auf den außeren Bereich der Querbohrung, so daß eine Kollision mit dem Bohrungsrand unvermeidbar ist. Der Bohrer wird bei der Kollision abgelenkt und berührt deshalb nicht den unteren Mantel der Probe, was sich mit den Beobachtungen im OP deckt.

Versuche mit in den Spalt eingebrachtem Gewebematerial ergaben keinen sichtbaren Unterschied in der resultierenden Schädigung und wurden im Sinne der besseren Reproduzierbarkeit verworfen. Da die erreichten geometrischen Abmessungen der erzeugten Kerbe von der Schärfe des verwendeten Werkzeugs abhängen, wurden die Bohrer je drei mal verwendet und dabei gleichmåßig ober die Proben verteilt. Das entspricht dem beobachteten durchschnittlichen Verschleiß von Operationsbohrern. Verwendet wurde eine Standard-OP-Preßluftbohrmaschine mit einem Moment von 2,5 Nm und einer Drehzahl von $17 \mathrm{~s}^{-1}$.

Die geschädigten Proben wurden im Anschluß mit $25 \%$, $30 \%$ und $40 \%$ des Durchnittes der zuvor ermittelten statischen Maximalbelastungen in einem Vier-PunktBiege-Schwingversuch mit 10 Herz getestet. Die resultierenden Momente lagen bei 9,3, 11,2 bzw. 14,5 $\mathrm{Nm}$. Die Proben wurden torsionsfrei zur Verringerung des Schubspannungseinflusses auf drehbaren Rollen gelagert (ASTM F383-73; DIN 54852; 1 SO 5833). Die Versuche wurden in Luft durchgefuhrt; der die Dauerfestigkeit verringernde Einflu $B$ der Korperflussigkeiten ist fur die untersuchten Werkstoffe gleich und beeintrăchtigt somit nicht die vergleichende Beurteilung des Materials untereinander.

\section{ERGEBNISSE}

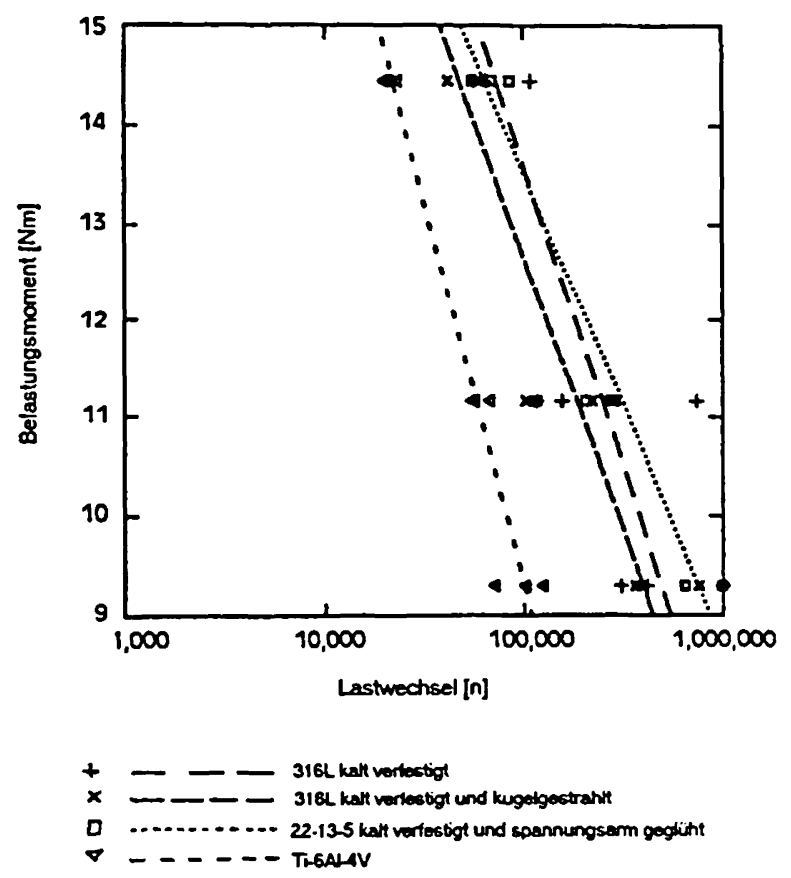

Abb. 2: Wöhler-Kurven der getesteten Werkstoffe

Wie bei der Voruntersuchung ungekerbter Proben [2], lassen sich die austenitischen Stăhle nicht sicher voneinander trennen. Ihre Festigkeit liegt unter der der ungekerbten Vergleichsproben. Alle Stähle quittieren die eingebrachte Kerbe mit einem ahnlichen, gemäßigten Festigkeitsverlust. Zweifellos läßt sich feststellen, daß Kugelstrahlen keine Verbesserung der Dauerfestigkeit erzielt.

Die untersuchte, furr Marknägel verwendete Titanlegierung liegt in ihrer Dauerschwingfestigkeit deutlich unter den anderen Werkstoffen. Da die Titanlegierung im unversehrten Zustand (außer bei hochsten Belastungen) den Stăhlen deutlich überlegen war, läßt das Verhalten nach der Schädigung durch den Bohrer (das ebenso bei starken Punzenkerben beobachtet werden konnte [2]) auf eine höhere Kerbempfindlichkeit schließen. Die Ermudungsgrenze war bei den Versuchsbedingungen uberschritten. Es ist also fragwürdig, ob die Vorteile von Ti-6Al-4V (wie zum Beispiel die höhere Elastizităt infolge geringeren Elastizitătsmodulus gegenüber austenitischen Stählen) dieses Risiko aufwiegen, wenn ein Material fur Marknägel gesucht wird. Schließlich seien die hoheren Kosten fur Titan-Implantate erwăhnt.

\section{LITERATUR}

[1] Robert W. Bucholz, Steven E. Ross, Kent L. Lawrence: „Fatigue Fracture of the Interlocking Nail in the Treatment of Fractures of the Distal Part of the Femoral Shaft." J. Bone and Joint Surgery 1987.

[2] C. Bourgeault, D. Loch, T. Yee, J. Bechtold, R. Kyle: „Can latrogenic Damage Reduce the Fatigue Life of Interlocked IM Rods?" Orthopaedic Transactions, J. Bone and Joint Surgery, 20(1):215-216,1996. 